\title{
LAMPI DI LUCE: DAI MILLISECONDI AGLI ATTO SECONDI ${ }^{1}$
}

\author{
ORAZIO SVELTO (*)
}

SunTO. - Da lungo tempo l'uomo ha usato impulsi di luce per studiare il movimento di oggetti sempre più veloci. Per quanto riguarda lo studio del movimento di oggetti macroscopici, si ricorda innanzitutto lo studio del galoppo del cavallo effettuato dal fotografo Eadweard Muybridge verso la fine dell'ottocento mediante un ingegnoso metodo basata su una serie di macchine fotografiche in cascata. Si riuscì così ad ottenere un film dell'intero moto del cavallo mediante apertura di obiettivi rapidi con durata dei milli secondi $\left(1 \mathrm{~ms}=10^{-3} \mathrm{~s}\right)$. Si ricorda anche l'introduzione delle lampade flash, avvenuta circa cinquant'anni dopo (1930), con le quali fu possibile fotografare il moto di oggetti ancora più piccoli e in moto molto più veloce (es. una pallottola dopo aver attraversato una mela oppure il movimento di un giocatore di golf mentre colpisce la relativa pallina). Con questa tecnica fu infatti possibile ottenere impulsi di luce della durata di circa un microsecondo $\left(1 \mu s=10^{-6} \mathrm{~s}\right)$. Ma è stato solo dopo l'invenzione del laser, avvenuta da parte di T.H. Maiman nel 1960, che fu possibile realizzare impulsi di luce di durata considerevolmente più breve. Utilizzando infatti laser a stato solido operanti nel così detto regime di mode-locking, è stato infatti possibile realizzare impulsi della durata di decine di picosecondi $\left(1 \mathrm{ps}=10^{-12} \mathrm{~s}\right)$ alla fine degli anni 70 , raggiungendo durate di pochi femtosecondi negli anni $90\left(1 \mathrm{fs}=10^{-15} \mathrm{~s}\right)$ fino a raggiungere $i$ valori attuali di circa 70 attosecondi $\left(1\right.$ as $\left.=10^{-18} \mathrm{~s}\right)$. In tutti questi anni dall'invenzione del laser, il gruppo di ricerca presso il Dipartimento di Fisica del Politecnico, guidato da chi scrive, è stato sempre all'avanguardia in campo internazionale nel realizzare impulsi sempre più brevi e nel loro utilizzo. Dopo l'introduzione del primo laser al Politecnico di Milano, avvenuto nel 1963 (cinquant'anni fa!), il gruppo riusciva a realizzare impulsi di soli 5 picosecondi nel 1969, un record internazionale per quel tempo. Lo stesso gruppo realizzava poi impulsi di soli 4,5 femtosecondi verso la fine degli anni 90, ancora un record per quel tempo, per poi ottenere impulsi di soli 130 attosecondi a metà degli

1 Questo articolo è particolarmente dedicato alla memoria del Prof. Emilio Gatti, recentemente scomparso, che ha rivestito un ruolo molto importante nell'indirizzare l'autore di questo lavoro alle discipline laser.

${ }^{(*)}$ Istituto Lombardo Accademia di Scienze e Lettere, Politecnico di Milano, Italia. E-mail: orazio.svelto@polimi.it 
anni 2000. Impulsi ultrabrevi di luce laser sono stati utilizzati, in tutti questi anni, da svariati gruppi in campo internazionale per lo studio del comportamento dinamico di molecole, di complessi molecolari e di sistemi nanoscopici a stato solido (quantum wells e quantum dots). A titolo di esempio, viene discusso un recente e importante lavoro effettuato dal gruppo del Politecnico sul comportamento dinamico del retinale della rodopsina, elemento primario della visione dell'occhio umano.

$* * *$

ABSTRACT. - Mankind has always been interested in studying the movement of ever faster objects upon using ever shorter light pulses. As a first example of a study of macroscopic samples, mention will be made about the study of a horse, while galloping, as obtained by the photographer Eadweard Muybridge around the end of years ' 800 . He was in fact able to obtain a complete recording of the horse movement upon using an ingenious system of several cameras, placed along the horse racing, equipped with shutters with a record opening time of about 2 millisecond $\left(1 \mathrm{~ms}=10^{-3} \mathrm{~s}\right)$. About 50 years later (1930) the introduction of flashlamps allowed the achievement of light pulses with duration of about one microsecond $\left(1 \mu \mathrm{s}=10^{-6} \mathrm{~s}\right)$. Upon using flashlamps in a repetitive mode, it was then possible to record the movement of much smaller and hence much faster objects (e.g. a bullet while crossing an apple or the fast movement of a golf player while hitting the golf ball). It was, however, only after the invention of the laser (T.H. Maiman, 1960) that people became able to generate much shorter light pulses. From initial pulse durations (middle of years '60) of about 10 picosecond ( 1 ps= $10^{-12} \mathrm{~s}$ ), one could then generate (during years ' 90 ) laser light pulses of a few femtoseconds $\left(1 \mathrm{fs}=10^{-15} \mathrm{~s}\right)$ down the present values of about 70 attoseconds $\left(1 \mathrm{as}=10^{-18} \mathrm{~s}\right)$. During all these years after laser invention, the research group at the Physics Department of Politecnico di Milano, leaded by the author of the present paper, has always been at the forefront of the international research to generate and utilize laser pulses of ever shorter duration. After the introduction of the first laser at the Politecnico di Milano at the beginning of 1963 (more than 50 years ago!), the research group was able to obtain light pulses of about 5 picoseconnds in 1969, a record value for that time. The same group then obtained record values of light pulses of about 4,5 femtoseconds, at the end of years '90, and of only 130 attoseconds around the middle of years 2000. Ultrashort laser pulses have been used, throughout all these years, by a large number of international groups to investigate the ultrafast dynamic behavior of molecules, molecular complexes, and solid state nanoscopic materials (such as quantum wells and quantum dots). As a representative example, some discussion will be presented about a recent work made by the research group of Politecnico about the retinal contained in the rodospin protein, a basic element for human vision.

\section{INTRODUZIONE}

Da lungo tempo l'uomo è stato affascinato dallo studio di oggetti in movimento così veloce da non poter essere percepito dall'occhio umano. Allo scopo ha spesso usato lampi di luce sempre più veloci. Tuttavia, prima dell'invenzione del laser avvenuta nel 1960, gli impulsi 
di luce più rapidi furono quelli disponibili dalle lampade flash, con durata degli impulsi tipicamente di circa un microsecondo $\left(1 \mathrm{~ms}=10^{-6} \mathrm{~s}\right)$ ma che poteva raggiungere, nei sistemi più avanzati, anche qualche nanosecondo $\left(1 \mu \mathrm{s}=10^{-9} \mathrm{~s}\right)$. Mediante lampade flash operanti a ripetizione, fu così possibile fotografare e seguire il moto veloce di oggetti sempre più piccoli (es. una pallottola in volo). Ma solo dopo l'invenzione del laser fu possibile realizzare impulsi di luce laser nettamente più brevi. Dalla durata iniziale, a metà degli anni '60, di circa 10 picosecondi (1 ps $\left.=10^{-12} \mathrm{~s}\right)$, si passò, negli anni ' 90 , a generare impulsi di pochi femtosecondi $\left(1 \mathrm{fs}=10^{-15} \mathrm{~s}\right)$ fino a raggiungere i valori attuali di circa 70 attosecondi ( 1 as $\left.=10^{-18} \mathrm{~s}\right)$.

In tutti questi anni dall'invenzione del laser, il gruppo di ricerca presso il Dipartimento di Fisica del Politecnico, guidato per lungo tempo da chi scrive, è sempre stato all'avanguardia in campo internazionale nel realizzare impulsi sempre più brevi e nel loro utilizzo.

In questo articolo, dopo un excursus storico sullo studio di movimenti rapidi prima dell'invenzione del laser, saranno descritti alcuni sviluppi principali nel realizzare impulsi di luce laser sempre più brevi e sarà esposto qualche esempio di utilizzo di impulsi così brevi nello studio di molecole di interesse chimico e biofisico.

\section{LO STUDIO DEL MOVIMENTO PRIMA DEL LASER}

Un primo esempio storicamente importante riguarda lo studio del galoppo di un cavallo. Esso aveva incuriosito molto Leland Stanford, fondatore della Central Pacific Railway, ottavo governatore della California e fondatore, nel 1891, della Leland Stanford Junior University, oggigiorno più comunemente chiamata Università di Stanford. Il suo amore per i cavalli, lo indusse infatti a porsi il seguente quesito: "Esiste un momento in cui un cavallo, al galoppo, tiene tutti e quattro gli zoccoli sollevati dal terreno?”. Non era infatti possibile risolvere questo quesito ad occhio nudo, data la (relativa) rapidità del moto. Egli pose pertanto questo quesito al noto fotografo inglese dell'epoca, Eadweard Muybridge, direttore del Photographic Survey del Governo degli Stati Uniti. Si dice che avesse promesso a Muybridge una ricompensa di ben $25.000,00 \$$ qualora costui fosse riuscito a rispondere al quesito.

Fu così che Muybridge, fra gli anni 1870 e 1880, si mise alacremente al lavoro per risolvere il problema. Da un lato, infatti, egli per- 
fezionò le lastre fotografiche allora disponibili, rendendole più sensibili alla luce. Dall'altro sviluppò un otturatore della macchina fotografica molto più rapido, con tempo di apertura di circa 2 millisecondi. Posizionò quindi 12 macchine fotografiche, così perfezionate, lungo il percorso del cavallo al galoppo, separate l'un l'altra da circa mezzo metro. L'apertura dell'interruttore di ciascuna macchina era poi comandata da sottili fili, posti in corrispondenza di ogni macchina fotografica in direzione ortogonale al percorso del cavallo e che il cavallo tagliava al suo passaggio azionando l'apertura del corrispondente otturatore. Un esempio in grado di rispondere al quesito di Leland Stanford è mostrato nella Fig. 1, dove sono indicati 8 fotogrammi consecutivi dei 12 realizzati. Da esso, in particolare dai fotogrammi 1-4, fu possibile a Muybridge di rispondere positivamente al quesito posto da Leland Stanford, aggiudicandosi il cospicuo premio. Da osservare, inoltre, che, dai successivi fotogrammi 5-8 è possibile anche studiare il modo in cui il cavallo, successivamente, "atterra" con le sue gambe sul terreno.



Fig. 1. Serie di immagini di un cavallo al galoppo, cosi come realizzate dal fotografo inglese Eadweard Muybridge.

Muybridge va pertanto considerato come il pioniere della fotografia (stroboscopica) ad alta velocità. Ma un avanzamento molto importante, per questo tipo di fotografia, fu realizzato con l'invenzione e lo sviluppo di lampade flash allo Xeno ad opera particolarmente dell'americano Harold Edgerton, Professore di Ingegneria Elettrica al Massachussets Institute of Technology. Operando soprattutto negli anni 1930-1950, egli infatti riuscì a realizzare sistemi di illuminazione mediante lampade flash in grado di realizzare impulsi di luce di durata 
breve fino a circa 0,3 microsecondi e intervallati fino a circa 1 microsecondo. Fu così possibile fissare il movimento di oggetti molto più veloci di un cavallo (ad esempio l'attraversamento di una mela da parte di una pallottola, Fig. 2a) oppure ottenere (mediante una serie ripetitiva di impulsi flash) la visualizzazione stroboscopica di un oggetto in rapido in movimento (ad esempio il movimento di un giocatore di golf per colpire, con la sua mazza, la pallina, Fig. 2b). Nella ricerca per realizzare impulsi di luce sempre più intensi e di breve durata, Edgerton arrivò, negli ultimi anni, a realizzare impulsi da lampade flash di durata di qualche nanosecondo $\left(1 \mathrm{~ns}=10^{-9} \mathrm{~s}\right)$.

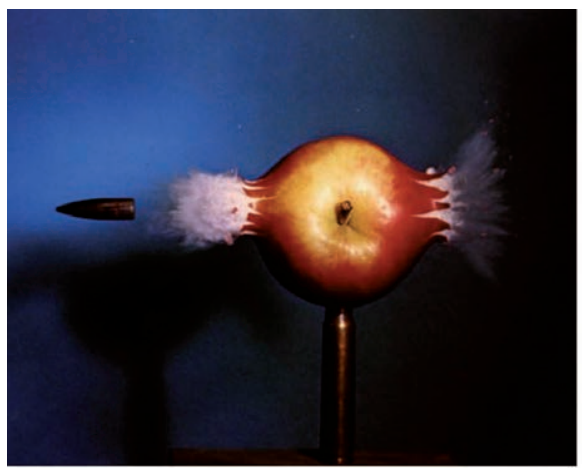

(a)

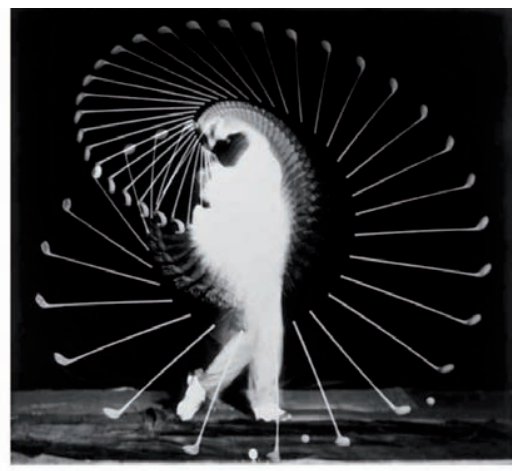

(b)

Fig. 2. (a) Istantanea di una pallottola mentre attraversa una mela;

(b) immagine stroboscopica di un giocatore di golf mentre muove la mazza da golf per colpire la pallina.

\section{LAMPI DI LUCE DOPO L'INVENZIONE DEL LASER}

La realizzazione di lampi di luce sempre più brevi e intensi subì una brusca accelerata con l'invenzione del Laser, avvenuta da parte di Theodore H. Maiman nel 1960 presso i Laboratori Hughes di Malibu (CA). Infatti, un paio di anni dopo questa invenzione, furono immediatamente realizzati, mediante la tecnica detta di Q-switching, impulsi di luce di alcuni nanosecondi. Pochi anni dopo (cioè nel 1965), mediante la tecnica detta di Mode-Locking, fu poi possibile realizzare impulsi di luce di alcuni (5-10) picosecondi. Successivamente, a partire dalla metà degli anni '80 e utilizzando nuovi laser a larga banda (a colorante e, 
soprattutto, a Titanio in zaffiro) e tecniche di compressione degli impulsi stessi, fu possibile realizzare impulsi laser con durata di alcuni (5-10) femtosecondi. Successivamente ancora, a partire circa dagli inizi degli anni 2000 ed utilizzando la tecnica di generazione di armoniche di ordine elevato, è stato possibile rompere la barriera del femtosecondo fino a generare impulsi di durata di alcune decine (67 è attualmente il valore record) di attosecondi. Più in dettaglio, la Fig. 3 mostra la durata minima degli impulsi laser realizzata nel tempo da vari gruppi internazionali interessati all'argomento, a partire circa da metà degli anni '60 fino ai giorni nostri (2010).

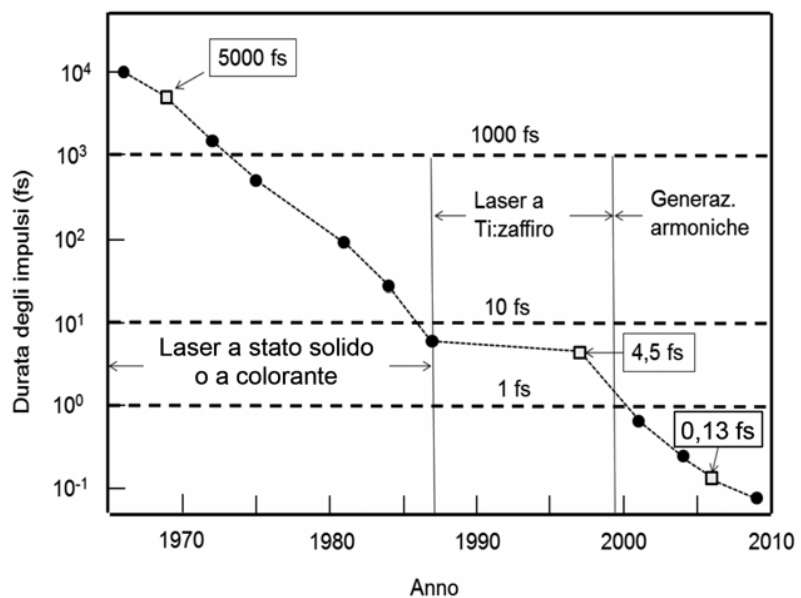

Fig. 3. Evoluzione, negli anni, della durata minima degli impulsi di luce realizzati mediante diversi tipi di laser e diverse tecniche.

Nella Fig. 3, appare opportuno notare che i punti indicati con un quadratino bianco (e col valore di durata indicato in un rettangolo) rappresentano altrettanti record realizzati dal gruppo laser del Politecnico di Milano, che chi scrive ha avuto l'onore di dirigere per oltre quarant'anni. Il primo valore, di $5.000 \mathrm{fs}$ ( 5 ps), fu realizzato nel 1969 mediante un laser a rubino operante in regime di mode-locking e con speciale configurazione della cavità risonante [1]. Un secondo valore record (4,5 fs) fu realizzato nel 1997 in collaborazione con ricercatori dell'Università di Vienna [2]. Per ottenere questo record si utilizzò un compressore di impulsi a fibra cava inventato dal gruppo 
del Politecnico di Milano l'anno precedente [3]. Fu così possibile comprimere gli impulsi di un laser di relativamente alta energia (1 $\mathrm{mJ}$ ), disponibile presso la citata Università, da $25 \mathrm{fs}$ al valore record citato. Il valore di 0,13 fs (130 as) fu quindi realizzato nel 2006, da ricercatori del gruppo del Politecnico, mediante la tecnica di generazione di armoniche di ordine elevato e utilizzando i singoli impulsi da circa $5 \mathrm{fs}$ realizzati in precedenza mediante la tecnica del compressore a fibra cava [4]. Da notare che questa fibra cava viene oggigiorno utilizzata da tutti i gruppi di ricerca in campo internazionale interessati a generare singoli impulsi ad attosecondi mediante la tecnica di generazione di armoniche di ordine elevato. Essa rappresenta dunque una delle invenzioni più importanti realizzate dal citato gruppo di ricerca del Politecnico.

Appare opportuno notare, a questo punto, che i valori record discussi in precedenza non sono stati qui brevemente discussi per indicare che il gruppo laser del Politecnico è andato negli anni continuamente a caccia di record, destinati peraltro come tutti i record ad essere rapidamente superati. La descrizione precedente intendeva, infatti, soltanto evidenziare che il gruppo si è costantemente mantenuto nelle posizioni più avanzate della ricerca in questo settore in tutto questo periodo! Per un testo dedicato alle celebrazione dei 150 anni del Politecnico stesso, appare dunque opportuno citare, nel seguito, una breve storia del gruppo stesso.

\section{Il laser al Politecnico di Milano}

La storia del Laser al Politecnico parte con l'invio di chi scrive presso l'Università di Stanford per un periodo di quasi due anni (19611963) con una borsa di studio del Consiglio Nazionale delle Ricerche (CNR). La borsa di studio fu assegnata dal CNR su proposta del Prof. Emilio Gatti, che aveva assegnato a chi scrive, nel 1959, una tesi di laurea su un argomento prodromo del laser. Si deve dunque alla lungimiranza di questo illustre Professore del Politecnico se chi scrive fu avviato al campo del laser fin dagli inizi di questa nuova disciplina. Al ritorno dagli USA (Febbraio 1963), presso l'allora Istituto di Fisica del Politecnico (ora Dipartimento di Fisica) fu quindi costituito un gruppo di ricerca costituito, oltre da chi scrive, dal compianto Prof. Carlo Alberto Sacchi e da due tecnici, gruppo finanziato interamente dal 
CNR stesso. Una foto di quel periodo (Fig. 4), mostra il primo laser realizzato al Politecnico (laser a Rubino) ${ }^{2}$. Di questa foto, in particolare, non si sa se rimarcare di più la giovane età dei partecipanti (incluso chi scrive che guarda in maniera assorta la traccia dell'azione laser nell'oscillografo) oppure la assoluta povertà della strumentazione disponibile (tavoli ottici in legno e banchi ottici reperiti nel magazzino della strumentazione usata per dimostrazioni agli studenti!). Tuttavia, con questa strumentazione così primitiva, fu possibile avviare una serie di ricerche d'avanguardia nel settore, che si imposero subito all'attenzione in campo internazionale. Di esse, particolarmente note ed apprezzate si dimostrarono le ricerche per realizzare impulsi di luce laser sempre più brevi per il loro utilizzo nello studio del comportamento ultra-rapido della materia.

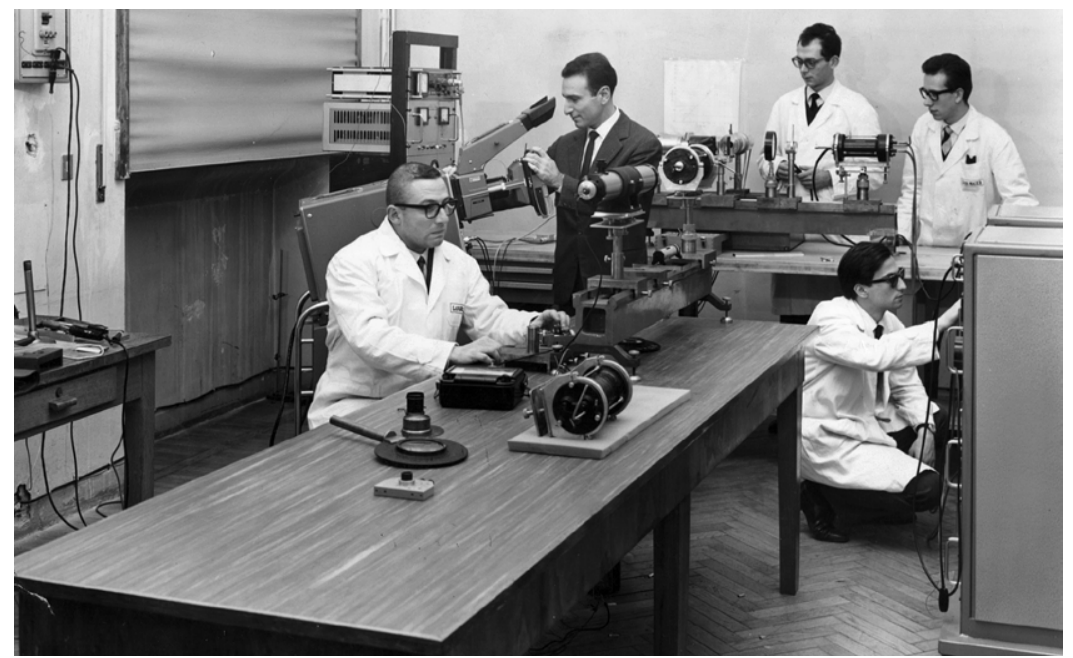

Fig. 4. Foto del primo laser al Politecnico di Milano (1963).

I successi del gruppo indussero quindi il CNR a istituire nel 1976, presso lo stesso Istituto, il Centro di Elettronica Quantistica e Strumentazione Elettronica (CEQSE) che chi scrive ha diretto ininter-

2 Da notare che l'anno 2013, oltre che marcare i 150 anni dalla fondazione del Politecnico di Milano, evento a cui il presente volume è dedicato, ha anche marcato i 50 anni del laser al Politecnico. 
rottamente per 25 anni, e cioè fino all'anno 2000. A quella data il CEQSE confluì in un nuovo Istituto allora istituito dal CNR (Istituto di Fotonica e Nanotecnologie), la cui sede è attualmente presso il citato Dipartimento di Fisica. In esso, con le sue varie sedi distaccate su tutto il territorio nazionale (6), attualmente operano circa 100 ricercatori equamente ripartiti fra personale universitario e personale CNR. Questo Istituto rappresenta ora, per ricerche nel campo dello sviluppo dei laser e delle relative applicazioni, il centro di ricerca più avanzato in campo Italiano e uno dei più avanzati in campo internazionale. Particolarmente importanti, in questo Istituto, sono le ricerche nel campo degli attosecondi, che hanno ricevuto lusinghieri successi in campo internazionale, ivi inclusi 4 cospicui grants dello European Research Council (ERC). A testimonianza di questa evoluzione, viene mostrata in Fig. 5 una foto del primo laboratorio ad attosecondi del Politecnico (1999). Esso fu realizzato mediante un finanziamento particolare dell'allora Istituto Nazionale di Fisica della Materia (INFM). In paragone alla foto originaria del 1963 (Fig. 4), in quest'ultima figura balza all'occhio, oltre che ovviamente la non più giovane età di chi scrive, la ricchezza della strumentazione ora disponibile (e ce ne 5, di questi laboratori, attualmente funzionanti).

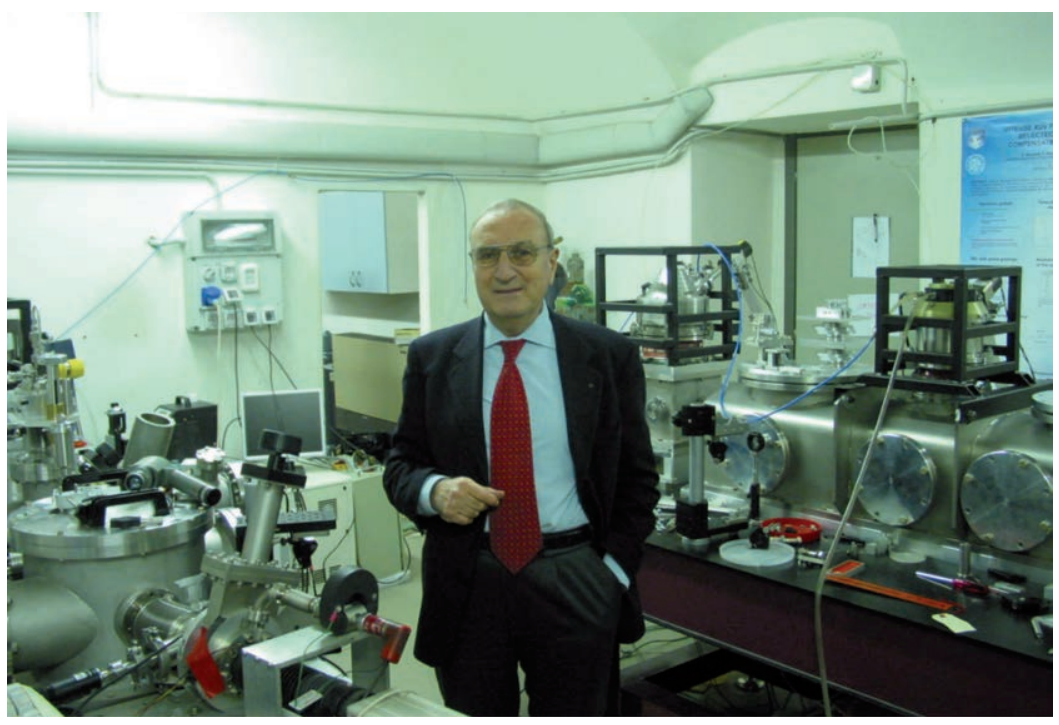

Fig. 5. Foto del primo laboratorio ad attosecondi del Politecnico di Milano (1999). 


\section{MisURA DI FENOMENI ULTRA-VELOCI}

Nei limiti di questo lavoro, è impossibile cercare di riassumere in maniera ancorché sommaria l'insieme di lavori che sono stati effettuati negli ultimi 50 anni sulle misure di fenomeni ultraveloci mediante laser a pico- e, successivamente, a femto-secondi. Basti al riguarda far presente che, ogni due anni, viene tenuto un congresso internazionale sull'argomento cui partecipano parecchie centinaia di ricercatori in campo internazionale, presentando alcune centinaia di lavori. A riguardo ci si limita a far riferimento all'ultimo atto di questi congressi ed ai lavori in esso contenuti [5]. A titolo di esempio, nel seguito ci si limiterà ad una discussione, molto sommaria, di uno studio particolarmente importante effettuato recentemente da ricercatori del gruppo di ricerca del Politecnico.

Come introduzione a questo lavoro, appare opportuno far presente che molti processi in natura coinvolgono l'assorbimento di luce, ad esempio il processo di visione del mondo animale e la fotosintesi nel mondo vegetale. In ambedue i casi si tratta di processi determinanti per l'esistenza del mondo animale e vegetale, rispettivamente. Questi processi, innescati dall'assorbimento di un quanto di luce, sono caratterizzati da eventi primari molto rapidi, con durata tipicamente inferiore al picosecondo. Una durata così breve dell'evento riduce infatti drasticamente la competizione dell'evento stesso con processi dissipativi nonradiativi, sempre presenti nel sistema, tipicamente più lenti e che tendono a ridurre l'efficienza del processo. Questo spiega la elevata efficienza di questi eventi primari che, in ultima analisi, rappresenta, nel processo evolutivo, un modo per garantire la sopravvivenza della specie. Ad esempio, animali con processi di visione molto efficaci hanno potuto più facilmente sopravvivere in ambiente di predatori, essi stessi caratterizzati da ottime capacità visive. Lo studio di questi processi ultraveloci risulta pertanto molto importante non solo per la comprensione intima dei relativi fenomeni ma anche per poter realizzare processi artificiali equivalenti (visione artificiale o fotosintesi artificiale). Per lo studio di questi fenomeni è necessario dunque eccitare il sistema con impulsi di luce ultra-brevi, e cioè con durata dai picosecondi in giù, e si è visto che solo il laser è in grado di generare questi lampi di luce.

Prima di mostrare un esempio di misura relativo al processo di visione, è opportuno discutere di quali tecniche, necessariamente ottiche data la rapidità dei fenomeni, vengano utilizzate per lo studio di questi 
fenomeni ultraveloci. Ce ne sono, ovviamente, parecchie ma la più comunemente usata è quella detta pump and probe. In questa tecnica, si irradia inizialmente il campione mediante un primo impulso di durata ultrabreve ed ad una lunghezza d'onda opportuna, $\lambda_{1}$, tale cioè da essere assorbita dal campione stesso. Questo primo impulso, detto impulso di pompa (pump), porta quindi il campione in stato eccitato. Un secondo impulso, a lunghezza d'onda generalmente inferiore $\lambda_{2}$, viene poi inviato sul campione ad un ritardo $\tau$ rispetto al primo impulso. I valori di $\lambda_{2}$ e di $\tau$ sono variabili da parte del ricercatore. Questo secondo impulso, detto di probe, esamina dunque lo stato del campione, al ritardo $\tau$, attraverso il processo di emissione stimolata (finché il campione si mantiene in stato eccitato), oppure attraverso il processo di assorbimento, qualora il campione effettui una transizione verso un nuovo stato fondamentale. Pertanto, lo studio dell'assorbimento del campione eccitato, ai vari ritardi e varie lunghezze d'onda, fornisce informazione sul cambiamento nel tempo del suo stato. Alternativamente, l'impulso di probe può essere costituito da uno spettro a larga banda in modo da misurare, ai vari ritardi, l'intero spettro di emissione stimolata o di assorbimento del campione, a seguito della sua evoluzione temporale.

\subsection{Dinamica dell'evento primario nel processo di visione}

Il meccanismo della visione, ad esempio nell'occhio umano, è descrivibile schematicamente mediante la Fig. 6. In essa è indicata un'immagine dell'occhio umano (Fig. $6 a$ ), nella cui retina sono contenuti i così detti coni (preposti alla visione, a colori, diurna) ed i bastoncelli (responsabili della visione, in bianco e nero, notturna) (Fig. 6b). Nei bastoncelli è poi contenuta la proteina detta rodopsina (Fig. 6c) che consiste di una sacca proteica (opsina) che contiene il cromoforo (retinale) responsabile del processo di assorbimento della luce (nei coni è invece presente la molecola di iodopsina, simile alla rodopsina e che contiene anch'essa, al suo interno, la molecola di retinale). La struttura della molecola retinale è mostrata in Fig. 7, in cui nella Fig. 7a è mostrata la configurazione in stato di riposo. Si tratta di una molecola complessa contenente doppi legami $-\mathrm{CH}=$ coniugati e quindi in grado di avere un forte assorbimento della luce incidente. Nella sua configurazione di riposo la molecola risulta ripiegata attorno alla posizione 11 dei legami coniugati (configurazione detta 11-cis). A seguito dell'assorbimento 
della luce, il retinale "raddrizza" i propri legami coniugati a partire dalla posizione 11, portandosi quindi nella configurazione di Fig. $7 b$ (detta alltrans). Si tratta dunque di un processo di isomerizzazione, in cui cioè la molecola cambia il suo stato conformazionale ma non cambia la sua struttura chimica (cambiando ad esempio il numero di atomi di carbonio presenti). Questa isomerizzazione comporta poi che la rodopsina (o la iodopsina) subisca dei complessi processi di modificazione proteica col risultato finale di liberare cariche elettriche responsabili della percezione visiva da parte del cervello. Pertanto, la isomerizzazione ultraveloce del retinale costituisce l'evento primario del meccanismo di visione, ad esempio dell'occhio umano. Il suo studio ha appassionato, negli anni, svariati ricercatori interessati a misure ultraveloci poiché, come misurato in passato da altri ricercatori, la isomerizazione della molecola di retinale avviene in circa $200 \mathrm{fs}$.

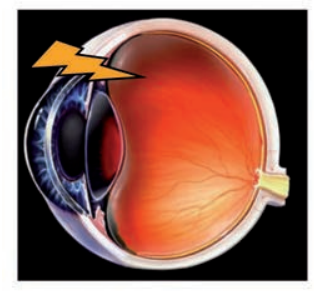

(a)

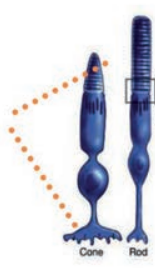

(b)

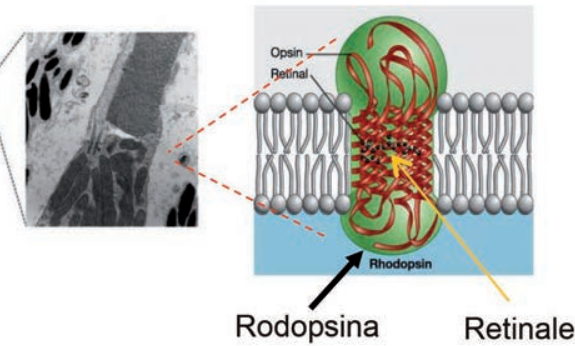

(c)

Fig. 6. Meccanismo della visione nell'occhio umano: (a) Struttura dell'occhio; (b) $i$ coni e $i$ bastoncelli responsabili della visione diurna (a colori) e notturna (in bianco e nero), rispettivamente; (c) la struttura della rodopsina, contenuta nei bastoncelli e che contiene al suo interno la molecola di retinale.

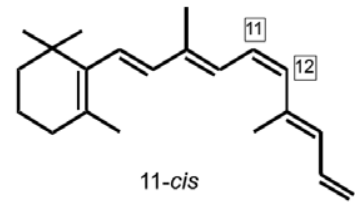

(a)

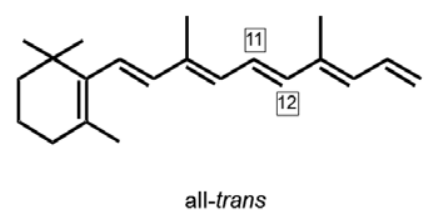

(b)

Fig. 7. Struttura della molecola di retinale (a) nella sua configurazione di base (11-cis), $e$ (b) nella configurazione in cui si porta a seguito dell'eccitazione luminosa (all-trans). 
Recentemente, il gruppo di ricerca del Politecnico è andato oltre questo dato globale misurando, ogni $20 \mathrm{fs}$, il cambiamento conformazionale della molecola durante il processo di isomerizzazione! [6]. La misura è stata effettuata mediante la tecnica di pump and probe e utilizzando, per il pump, impulsi di durata pari a 10 fs alla lunghezza d'onda di $500 \mathrm{~nm}$. Per il probe sono stati poi usati, per coprire l'intero intervallo di variazione dinamica del retinale, sia impulsi con durata di 7 fs e con banda da 500 a $700 \mathrm{~nm}$ (visibile) che impulsi da 13 fs e con banda da 820 a $1.050 \mathrm{~nm}$ (infrarosso). Il risultato complessivo di questo insieme di misure è rappresentabile come indicato in Fig. 8. In esso si vede che la molecola di retinale, una volta eccitato dall'impulso di pompa, si porta in uno stato energetico dello stato $S_{1}$ del retinale 11-cis (stato indicato con una prima curva a campana verde nella figura).

Una volta portato in questo stato, il retinale evolve verso il minimo di energia del suo stato eccitato $S_{1}$ in circa $50 \mathrm{fs}$. Durante questo periodo è stato osservato che l'impulso di probe viene amplificato nel passaggio attraverso il campione a causa del processo di emissione stimolata dallo stato $S_{1}$ allo stato $S_{0}$ (righe frecciate in blue e che puntano verso il basso, nella figura). A causa della conformazione degli stati $\mathrm{S}_{1}$ ed $\mathrm{S}_{0}$, si vede dalla figura che la lunghezza d'onda dell'emissione stimolata cresce con l'aumentare del ritardo dell'impulso di probe. Dopo circa 50 fs il retinale effettua una transizione verso uno stato energetico relativo al secondo minimo dello stato $\mathrm{S}_{0}$ (retinale all-trans, eccitato). Successivamente, in circa $100 \mathrm{fs}$, il retinale all-trans eccitato si porta verso il secondo minimo di energia del suo stato fondamentale $\mathrm{S}_{0}$ (retinale all-trans non eccitato). Durante questa transizione, è stato osservato nell'esperimento citato che l'impulso di probe viene attenuato nel passaggio attraverso il campione a causa del processo di assorbimento dallo stato $\mathrm{S}_{0}$ allo stato $\mathrm{S}_{1}$ (righe frecciate in rosso e che puntano verso l'alto, nella figura). In questo caso, a causa ancora della conformazione degli stati $S_{0}$ ed $S_{1}$, la lunghezza d'onda dell'assorbimento decresce con l'aumentare del ritardo dell'impulso di probe. Alla fine questo secondo processo, il retinale si trova nello stato fondamentale della molecola all-trans (il suo stato è indicato ancora con una seconda curva a campana di colore verde).

In conclusione di questo discorso, si può dire che il citato gruppo è riuscito a effettuare una serie di fotogrammi (spettrali) relativi alla dinamica del retinale, di emissione stimolata o di assorbimento, con potere risolutivo di ciascun fotogramma pari alla durata degli impulsi usati e quindi di circa $10 \mathrm{fs}\left(1 \times 10^{-14} \mathrm{~s}\right)$. Per paragone, è opportuno 
ricordare che il potere risolutivo temporale, realizzato dai fotogrammi di Muybridge per lo studio del galoppo del cavallo e citato come punto di partenza della nostra esposizione, era di circa $2 \mathrm{~ms}\left(2 \times 10^{-3} \mathrm{~s}\right)$, tempo cioè 200 miliardi di volte maggiore.

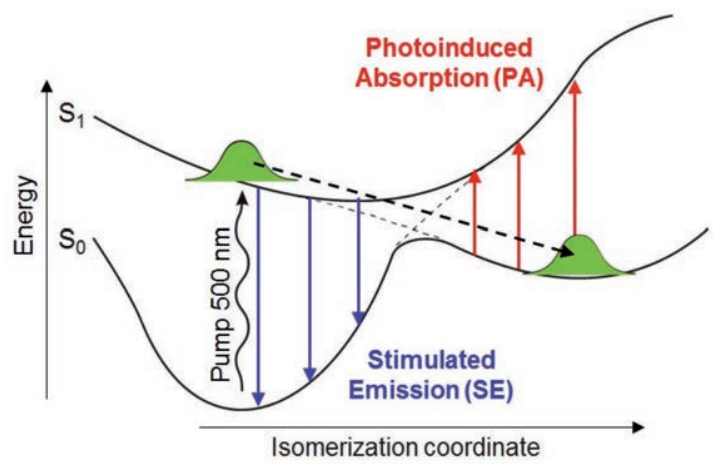

Fig. 8. Descrizione complessiva del processo della dinamica del retinale, cosi come ottenuta nel riferimento [6] mediante tecniche molto sofisticate di pump and probe.

\section{LA SCIENZA DEGLI ATTOSECONDI}

Da alcuni anni, in qualche decina di laboratori d'avanguardia in campo internazionale è stato possibile realizzare impulsi laser con durata intorno a 100 as. Si è così aperto un nuovo campo della scienza che va appunto sotto il nome di "Scienza degli Attosecondi". In generale si può dire che mentre lo studio dei fenomeni ultraveloci mediante impulsi a femto-secondi ha riguardato il moto degli atomi in molecole complesse o il trasferimento di energia fra molecole, lo studio dei fenomeni ultraveloci mediante impulsi ad attosecondi (cioè la scienza degli attosecondi) mira a studiare il moto degli elettroni all'interno di una singola molecola, una volta portati fuori dalla loro posizione di equilibrio. Si è aperta dunque una nuova frontiera della Fisica, della Chimica e della Biologia che ha a che fare col moto degli elettroni eccitati, moto di per sé intrinsecamente molto più rapido di quello dei singoli atomi di una molecola. Da notare che gli elettroni più esterni di un sistema molecolare formano i legami necessari fra $i$ vari atomi del sistema e che quindi il moto degli elettroni costituisce la base di ogni reazione chimica o biochimica. 
E' ancora tuttavia presto per giudicare la reale portata di questo nuovo campo della Fisica né appare opportuno presentare qualche risultato particolarmente rilevante. Per ulteriori dettagli sulle potenzialità di questo campo e sugli esperimenti iniziali si rimanda pertanto ad un rappresentativo lavoro di rassegna [7].

\section{Conclusioni e Prospettive}

In conclusione, si può dire che l'uomo è sempre stato affascinato dalla possibilità di studiare, mediante lampi di luce sufficientemente rapidi, il moto di corpi via via più piccoli e quindi intrinsecamente più veloci. Così, prima dell'invenzione del laser, la durata dei lampi di luce è andata via via decrescendo, in un arco di tempo di circa 100 anni (da metà '800 a metà '900), da qualche millisecondo a qualche nanosecondo. Con questi impulsi è stato possibile visualizzare e quindi studiare il moto di oggetti macroscopici in moto sempre più rapido (ad esempio dal cavallo ad una pallottola). Dopo l'invenzione del laser (1960), la durata dei lampi di luce laser, nel giro di qualche anno, è scesa rapidamente da qualche nanosecondo ad alcuni picosecondi. Successivamente, durante questi ultimi 50 anni circa (1965-2016), la durata degli impulsi di luce laser è gradualmente scesa da alcuni picosecondi a qualche decina di attosecondi. Con questi impulsi è stato possibile studiare la dinamica degli atomi di sistemi nanoscopici (dalle molecole semplici a complessi molecolari) con dinamica via via sempre più rapida. Con l'avvento degli impulsi ad attosecondi si è aperta ora una nuova era che ha a che fare non più con la dinamica di atomi in complessi molecolari ma con quella degli elettroni stessi di questi complessi.

E' ancora presto per giudicare le prospettive di questa nuova scienza (la scienza degli attosecondi) ma le sorprese maggiori devono forse ancora arrivare.

\section{RINGRAZIAMENTI}

L'autore desidera ringraziare i colleghi e collaboratori Giulio Cerullo, Sandro DeSilvestri e Mauro Nisoli per l'aiuto fornito durante la stesura di questo manoscritto. 


\section{RIFERIMENTI BIBLIOGRAFICI}

[1] R. Cubeddu, R. Polloni, C.A. Sacchi, O. Svelto. Picosecond Pulses, TEM00 Mode, Mode-Locked Ruby Laser. IEEE J. Quant. Electr.QE-5, 470 (1969).

[2] M. Nisoli, S. De Silvestri, O. Svelto, R. Szipocs, K. Ferencz, Ch. Spielmann, S. Sartania, F. Krausz. Compression of High Energy Laser Pulses below 5 fs. Opt. Letters, 22, 522-524 (1997).

[3] M. Nisoli, S. De Silvestri, O. Svelto. Generation of High Energy 10 fs Pulses by a New Pulse Compression Technique. Appl. Phys. Letters, 68, 2793-2795 (1996).

[4] G. Sansone, E. Benedetti, F. Calegari, C. Vozzi, L. Avaldi, R. Flammini, L. Poletto, P. Villoresi, C. Altucci, R. Velotta, S. Stagira, S. De Silvestri, M. Nisoli. Isolated Single-Cycle Attosecond Pulses. Science 314, 443 (2006).

[5] Ultrafast Phenomena XIX. Atti del XIX Congresso Internazionale, Okinawa, Giappone, 7-11 Luglio 2014. Editori: K. Yamanouchi, S. Cundiff, D. de VivieRiedle, M. Kuwata-Gonokami, L. DiMauro. Springer Verlag, New York, Vol. 162 (2015).

[6] D. Polli, P. Altoè, O. Weingart, K.M. Spillane, C. Manzoni, D. Brida, G. Tomasello, G. Orlandi, P. Kukura, R.A. Mathies, M. Garavelli, G. Cerullo. Conical intersection dynamics of the primary photoisomerization event in vision. Nature 467, 440 (2010).

[7] F. Krausz, M. Ivanov. Attosecond Physics. Rev. Modern Phys. 81, 163 (2009). 